Faculty of Science

Faculty Publications

This is a post-review version of the following article:

The past, present and potential for microfluidic reactor technology in chemical synthesis

Katherine S. Elvira, Xavier Casadevall i Solvas, Robert C. R. Wootton and Andrew J. deMello

2013

The final published version of this article can be found at:

https://doi.org/10.1038/nchem.1753

Citation for this paper:

Elvira, K.S., Casadevall I Solvas, X. Wootton, R.C.R. \& deMello, A.J. (2013). The past, present and potential for microfluidic reactor technology in chemical synthesis. Nature Chemistry, 5, 905-915. https://doi.org/10.1038/nchem.1753 


\section{The past, present and potential for microfluidic reactor technology in chemical synthesis}

5 Katherine S. Elvira, ${ }^{*}$ Xavier Casadevall i Solvas, Robert C. R. Wootton and Andrew J. deMello*

Department of Chemistry and Applied Biosciences, Institute for Chemical and Bioengineering, ETH Zürich,

(*katherine.elvira@chem.ethz.ch and*andrew.demello@chem.ethz.ch)

15 The past two decades have seen far-reaching progress in the development of microfluidic systems for use in the chemical and biological sciences. Herein we assess the utility of microfluidic reactor technology as an essential tool in chemical synthesis in both academic research and industrial applications. We highlight the successes and failures of past research in the field and provide a catalogue of chemistries performed in a microfluidic reactor. Subsequently, we assess the current roadblocks hindering the widespread use of microfluidic reactors from the perspective of both synthetic chemistry and industrial application. Finally, we will set out seven challenges that we hope will inspire the future research in this field. 
The advent of microfluidic technology as a basic tool for chemical synthesis is slowly coming of age, particularly in industrial settings. As with many new techniques, initial claims regarding performance, uniqueness and applicability were optimistic and wide ranging. The field has now had time to mature and it is time to look back on some of these early claims and compare them to actual progress, with a view to

30 predicting how and if microfluidic reactors can reach their full potential in the field of chemical research.

Microfluidic systems manipulate and control fluids that are geometrically constrained within environments having internal dimensions, or hydrodynamic diameters, most easily measured in microns. Although the first example of a microfluidic device can be traced back to $1940,{ }^{1}$ work by Terry and co-

35 workers describing the fabrication and testing of a chip-based gas chromatograph, ${ }^{2}$ and later studies by Manz $^{3}$, Mathies $^{4}$ and others in the early 1990s in the field of chip-based separations, better define the origins of mainstream microfluidic total analysis systems ( $\mu$ TAS) as they are understood today. At a similar time, the field of microfluidic reaction technology for chemical synthesis was developing quickly with notable contributions from researchers at GlaxoSmithKline (UK), ${ }^{5}$ Massachusetts Institute of Technology

40 (USA), ${ }^{6}$ the Institut für Mikrotechnik Mainz (Germany) ${ }^{7}$ and Imperial College London (UK), ${ }^{8}$ amongst others.

The intrinsic advantages associated with performing chemistry in microfluidic devices have been discussed extensively elsewhere, but in simple terms are due to the scale-dependent processes of heat and mass

45 transfer. ${ }^{9}$ Small fluidic volumes dictate that low Reynolds number regimes are the norm, with fluids being increasingly influenced by viscosity rather than inertia. In addition, large surface area-to-volume ratios ensure thermal homogeneity across the reactor and rapid heat transfer between the device and the contained fluid. These basic properties give rise to the following broad advantages that have made the technology attractive (in principle) for chemical synthesis in both industry and academia. 
Small reagent volumes. There is a distinct cost advantage in using small volumes of precious reagents where minimal amounts may be available for testing or processing. Particularly when used for informatics rather than for product synthesis, microfluidic reactors consume far less reagent than bulk systems to gather the same, and in most cases more chemical information. ${ }^{10,11}$

55 Selectivity. Many chemical and biological reactions generate more than one product from the same reactant pool depending on the local conditions. This phenomenon is often ascribed to kinetic versus thermodynamic control of the reaction pathway. ${ }^{12}$ In microfluidic reactors the degree of control over local conditions is such that it is often possible to select one product over another with high precision. ${ }^{13}$

Green credentials. Since heat transfer becomes more efficient as reaction volumes shrink, the amount of

60 energy consumed per unit temperature rise can be made extremely small, leading to significant environmental benefits. ${ }^{14}$ Similarly the improved selectivity ${ }^{15}$ mentioned above leads to less stringent reaction clean-up, and thus simpler chemistry than is typical on the macroscale.

Rapid reactions. It has often been claimed that microfluidic reactors produce "faster reactions" than those performed on the macroscale. ${ }^{16}$ Specifically, space-time yields (product formed per reactor volume 65 and time) in microfluidic reactors are consistently reported to be higher than those in bulk reactors. ${ }^{17}$ It is, however, difficult to make direct comparisons, as bulk reactions are rarely optimised to terminate exactly at the final equilibrium position of the reaction (reaction completion or endpoint), but often incorporate extra time to ensure completion has been reached. Reactions performed in microfluidic reactors are rarely run for longer than the time required to reach the reaction endpoint, since they can be closely

70 monitored to determine reaction completion. Accordingly, reaction times from literature syntheses are almost always incompatible with times associated with microfluidic formats. It is also important to note that there is no reason to expect the intrinsic rate of rate-limited reactions to increase within a microfluidic device compared to a similar "bulk" reaction unless a wall-mediated mechanism is invoked. This is simply because reaction kinetics do not rely on large-scale diffusion. Despite this issue, the rate of 75 mass-transfer limited reactions (i.e. reactions operating in the diffusive domain) will typically be increased 
by the small characteristic dimensions of microfluidic reactors, since in mass-transfer limited systems diffusive effects are significant with respect to the overall rate. ${ }^{18}$

Small footprints. Flow reactors almost always have a smaller footprint per kilogram of product than macroscale reactors. ${ }^{19}$ Adoption of microfluidic formats allows this scale to be further reduced since more 80 efficient heat transfer means that requirements for heat exchange equipment are minimised. ${ }^{20}$

Safe reactions. Microfluidic reactors have several intrinsic properties that make them safe environments in which to perform dangerous chemistry. Firstly, the small instantaneous volumes involved mean that reactions involving reactive, toxic or explosive intermediates can be carried out in safety. ${ }^{21}$ Furthermore, the high surface area-to-volume ratios within the channel allow the rapid transport of heat when 85 performing exothermic reactions ${ }^{22}$ and the enhancement of wall quenching in radical mediated systems. ${ }^{23}$

That said, scientists are not prophets. The predictions made and the hype generated around early stage technology is often wide of the mark when examined with the benefit of hindsight. Accordingly, some of the initial predictions relating to the development of chemical microfluidic systems bear inspection. Below 90 are a few of the standout predictions that have been made, or questions that have been posed, around microfluidic reaction technology, together with the current answer to these questions.

Will microfluidic technology replace the flask? This was a question posed often enough to become a review title. ${ }^{24}$ The notion was that a microfluidic reaction system could be used for as wide a range of 95 chemical operations as the venerable round-bottomed flask. To be fair, most people asking this question came to the same conclusion: there are areas where the application of microfluidic reaction technology makes sense, and others where the flask will remain the modality of choice.

Numbering up not scaling up..$^{25}$ It has long been suggested that the way to transform microfluidic 100 chemistry into an industrial tool is to massively parallelise the reactors (numbering up or scaling out) 
rather than to increase the characteristic dimension of the channel (scaling up). There have been numerous examples of both approaches using microfluidic reaction technology. As with most things a balance of factors must be considered. Scale out requires complex flow distribution and elaborate reaction monitoring methods, ${ }^{26}$ whereas scale up, as we will show, gives a limited accessible reaction phase space that is comparable to microfluidic reactor performance.

Point of use production. ${ }^{27}$ It has been suggested that chemicals, particularly drugs, could be manufactured in miniature factories at the point of use rather than in large central facilities. In some cases this has been successfully accomplished, ${ }^{28}$ but is best applied to the production of high value materials with limited 110 shelf lives.

The product of microfluidic synthesis will be information. Given the low volumetric throughput of individual microfluidic channels, but the rapid throughput of reaction conditions within the same channels, it has been argued that the true product from microfluidic chemistry will be chemical

115 information. ${ }^{8,29}$ This has certainly proven to be the case given the interest in reaction optimisation systems and discovery flow systems in general, as will be discussed below. Whether this is the main use of microfluidic reaction technology remains to be seen, since this may represent the low hanging fruit in terms of applying laboratory methodology commercially.

120 In the remaining discussion, we will examine how microfluidic chemistry has performed relative to these initial predictions and set out some of the challenges that still prevent the technology reaching its full potential. For the interested reader, Tables 1 and 2 in the Supplementary Information provide a comprehensive directory of chemical and nanomaterial syntheses performed in microfluidic reactors. This list is not exhaustive, but contains pertinent and representative references for each reaction, which 125 highlight the wealth of synthetic reactions that have been undertaken in microfluidic environments. 
Against this backdrop, we focus on two additional research areas currently driving microfluidic reactor technology, namely industrial research and reaction optimisation. We will then highlight the current barriers that are detrimental to microfluidic reactor research and we will conclude by posing seven challenges that aim to inspire research crucial to the future of microfluidic reactor technology.

\section{Microfluidic Reactors in Industry}

Although a significant proportion of microfluidic reactor research has been performed in academia, industry has also taken an inquisitive interest in this technology. To a certain extent, successful

135 microfluidic reactor chemical research has inevitably become linked to industry, inasmuch as the industrial adoption of academic research provides a direct measure of the commercial importance of microfluidic technology and its relevance to the wider world. Generally, it has been in the context of continuous flow synthesis that microfluidic reactor technology is attractive to industry, due to inherent properties such as cost reduction, efficient heat transfer and rapid mixing. ${ }^{30-32}$ In addition, the enhanced

140 control of reaction conditions and ability to operate at high pressures, ${ }^{32}$ combined with the safety advantages of small volume usage, ${ }^{33}$ ease of scale out ${ }^{31}$ and their capability for continuous ${ }^{34}$ and integrated operation have made their adoption desirable. In fact, an extensive assessment on a range of reactions performed in both microfluidic and conventional reactors concludes that in most cases there is a marked decrease in the residence time of the reaction and an increase in selectivity and conversion when 145 synthesis is performed in microfluidic reactors. ${ }^{35}$

Gaining a thorough understanding of the industrial perspective on microfluidic reactor technology is however complicated since most research is not published in peer-reviewed literature, but as patents. To this end, Hessel and co-workers have provided a comprehensive review of patents in microfluidic reactors 150 and microprocess engineering prior to $2006^{36}$ and from then until $2010 .{ }^{37}$ In their first review, they note 
that much of literature focused on cost-effective mass production rather than the development of microfabrication techniques, ${ }^{36}$ highlighting a potential conflict of interest between academia and industry. In their most recent review, they predict that microfluidic reactor patents will increasingly focus on structures and modes of operation for specific reactions. ${ }^{37}$ Lonza has been one of the most active players

155 in the field of microfluidic reactor technology, and their papers and patents provide a representative guide to the development of such technology in an industrial environment. Lonza's core research interests in this area include gas-liquid reactions, ${ }^{38}$ specific syntheses (such as nitration and hydrogenations), ${ }^{31,39}$ multi-injection techniques for staggered reagent dosing ${ }^{40}$ and investigating flow, ${ }^{41}$ pressure drops and mixing ${ }^{42}$ properties of microfluidic reactors. More in-depth discussions on other industrial activities are 160 provided elsewhere. ${ }^{43,44}$ Today, the applications of microfluidic reactor of most interest to industry include bulk and fine chemistry, organic, inorganic and polymer particle synthesis, pigments, emulsions, microencapsulates and steam reforming. These, and others, have been recently reviewed in a threevolume book series. ${ }^{45}$

165 To transfer technology from an academic environment to the industrial realm, the initial technology must be scalable, ${ }^{34}$ have integrated catalysts if needed, ${ }^{46}$ a high production capacity and integrated flow process, ${ }^{47}$ robust designs and, in compliance with the capitalist post-industrial revolution philosophy, have low man-power requirements and low initial investment. ${ }^{47}$ Industrial research has developed certain techniques that aim to produce robust and flexible microfluidic reactors with industrial scale production

170 capacities. For example, the use of microfluidic reactors with modular designs for scale out and flexibility in end use is common. ${ }^{30}$ Ease of scale out is a major benefit of microfluidic reactors over traditional batch production techniques. Since the dimensions of each reactor do not change, the chemistry taking place does not change either, ${ }^{48}$ and the same reactor can be used both for lab-based developmental research and industrial production. ${ }^{49}$ 
The use of commercially available microfluidic reactors in laboratories for academic research is a recent and much overlooked development in this area. Researchers can use off-the-shelf systems to mimic industry conditions and facilitate the transition between academic research and industrial production. The commercial availability of microfluidic systems is significant because it enables more researchers to access

180 the technology without having to master, or have access to, the often elaborate microfabrication techniques used in chip manufacture. These microfluidic reactors are robust and designed such that multiple syntheses can be developed in what effectively becomes one module in a scale out system. For example, the Uniqsis FlowSyn microfluidic reactor has been used for scaling out a diazotransfer reaction ${ }^{50}$ and Lonza have developed the FlowPlate microfluidic reactor for lab use outside industrial settings (Figure

185 1). Other companies with microfluidic reactor lab-based products include Alfa Laval, $^{51}$ Corning, $^{52}$ Microinnova, ${ }^{53}$ Chemtrix, ${ }^{54}$ Syrris ${ }^{54}$ and FutureChemistry. ${ }^{55}$

As noted, a key area where microfluidic reactor characteristics complement industrial requirements is in green chemistry. Green chemistry and engineering are attractive to industry ${ }^{33}$ due to the cost related

190 advantages and sustainability supplied. ${ }^{32}$ In simple terms, green industrial chemistry aims to decrease the energy requirements associated with chemical synthesis, increase throughput per unit production area, decrease reagent consumption and use less hazardous chemicals, all of which are inherent to microfluidic reactor systems. The use of microfluidic reactors can also open up new synthetic routes for industry, especially where the hazards associated with the reaction are greatly reduced. To this end, Kralisch et al.

195 recently performed a life cycle assessment of the advantages of performing the two-step conversion of $m$ bromoanisole into $m$-anisaldehyde in commercially available continuous microscale reactors versus commercially available macroscale, semi-continuous, batch reactors. ${ }^{56}$ This reaction is notoriously hard to scale up due to its highly exothermic nature, and because the yield typically decreases with reactor size. The authors found that the advantages of performing the reaction in Cytos stainless steel microfluidic 200 reactors stemmed mostly from their improved heat exchange capabilities. In fact, it was possible to perform the synthesis at a higher temperature and to therefore dispense with the cryogenic systems 
required on the macroscale. Other research has show that it is possible to use multi-injection of reagents into channels to provide a semi-batch approach to synthesis, whilst still operating in a continuous flow regime. ${ }^{40}$ This enhances temperature control and benefits reactions where hot-spot formation causes low

205 yields due to the fast and highly exothermic nature of the reaction itself. ${ }^{57}$ Another technique adopted by industry to enhance device robustness, and hence sustainability, is the use of arrays of parallel channels rather than a single long channe $\left.\right|^{58}$ to prevent pressure loss in the system. Finally, plate-stack reactors are also used, where different volumes within the reactor are used to increase residence times, increase the speed of mixing, enhance heat exchange and minimise pressure drops. ${ }^{34}$

A major driving force for the industrial adoption of microfluidic reactors is their use in situations where the proposed reaction is not otherwise possible. With this in mind, Chambers and Spink developed microfluidic reactors for selective fluorination and perfluorination of organic compounds ${ }^{59}$ and demonstrated efficient reaction scale out. ${ }^{60}$ Another striking example is the development of a microfluidic

215 reactor for on-site and on-demand synthesis of positron emission tomography (PET) tracers. ${ }^{28}$ Recent work by GE Healthcare for example, has focused on investigating device geometry for the reduction of autoradiolysis in polyether ether ketone (PEEK) reactors. ${ }^{61}$ An assessment of microfluidic tools for the synthesis of PET tracers can be found elsewhere, ${ }^{62}$ whilst a laboratory based microfluidic reactor for the synthesis of the radiopharmaceutical 2-deoxy-2-[ $\left[{ }^{18} \mathrm{~F}\right]$ fluoro-D-glucose $\left(\left[{ }^{18} \mathrm{~F}\right] \mathrm{FDG}\right)$ can be seen in Figure 2 .

In conclusion, a review of the patent and paper literature published by industrial researchers suggests that microfluidic reactors are commonly viewed as an enabling technology, rather than as a replacement for current techniques. A significant portion of reported research has focused on the miniaturisation of hazardous reactions ${ }^{33}$ and those where safety is the major concern. ${ }^{63}$ This research goes hand in hand 225 with the concept of using engineering, in this case microfluidic reactors, to bypass limitations of specific reactions or restrictions provided by process conditions, ${ }^{64}$ such as safety hazards or harsh temperature and pressure conditions. As off-the-shelf microfluidic reactor technology becomes more common in 
academic research laboratories, a close relationship between academic research and industrial application will also develop, where academic research is performed in systems easily scaled for industrial production.

230 Accordingly, characteristics such as economic viability, scale out potential, production capabilities, temperature control, reactor flexibility and reaction kinetics should be considered at the initial stages of experimental design.

\section{Reaction Optimisation}

As previously discussed, at an early stage in the development of microfluidic reaction technology it was suggested that the true product from microfluidic reaction systems would be information, rather than a more tangible substance or intermediate. ${ }^{65}$ Not surprisingly, there has been a great deal of work on microfluidic reaction optimisation. ${ }^{66}$ Low instantaneous volumes (due to the small reactor volumes) mean

240 that individual sets of reaction conditions can be screened rapidly in comparison to batch (or robotic) systems. An example of a microfluidic reactor used for reaction optimisation is shown in Figure 3 . In this work, the reaction phase space relating to temperature, reagent concentration and residence time for glycosylation reactions was rapidly sampled to determine the optimum reaction conditions. Figure 4 shows another example of reaction optimisation performed in a microfluidic reactor. In this case multitrajectory optimisation strategies and on-line reaction monitoring were used to intelligently find the optimum reaction conditions for a Paal-Knorr synthesis.

Much microfluidic reactor research has been carried out in industrial settings to guide larger flow systems for production. ${ }^{47}$ Indeed, there is a strong enough concordance between reaction phase spaces on the 250 micrometre and millimetre scales to allow a relatively smooth transition between low volumetric throughput discovery systems and higher volumetric throughput production systems. ${ }^{29}$ This equivalency of "micro" and "meso" reactors can be theoretically tested by looking at the characteristic dimensionless 
numbers associated with the reactor fluid flow (Figure 5). In this example, and for the purposes of equivalency, an arbitrary reactor length of 5 metres is assigned and the residence time is used as a scale-

255 invariant proxy for flow rate. From Figure 5 it can be clearly seen that for a wide range of residence times, a $500 \mu \mathrm{m}$ or $1 \mathrm{~mm}$ diameter reactor channel operates within this microchannel-like zone of equivalency for a range of residence times. It should, however, be noted that not all dimensionless numbers, and thus not all aspects of fluid behaviour, scale in an identical fashion. In particular, mixing and thermal diffusion (best characterised by Fourier numbers) and convection or buoyancy effects (governed by Grashof or

260 Rayleigh numbers) scale non-linearly with the characteristic dimension (an explanation is provided in the Supplementary Information). Therefore, if these phenomena are to be controlled the correct scale of reactor is absolutely critical.

Although this "micro" to "milli" scale conversion is a strategic use of microfluidic reaction technology, it is 265 simply an extension of the existing discovery/scale up paradigm (where a reaction is investigated on a small scale and the size of the reaction vessel is progressively scaled up to production scale, with concomitant variations in the chemistry) into a flow format. For microfluidic reaction technology to perform at its best, the technology must enable a process to be performed in a manner inaccessible by traditional means. Intelligent synthesis is a concept that strives to do just that. The continuous flow nature 270 of microfluidic reaction systems ensures that reaction conditions such as temperature, reaction time and reagent concentration can be varied, and the resulting changes in product ratios and yields recorded, in close to real time. This enables the production of high-end products where the product specification is of primary importance. A typical example of this would be in the production of compound semiconductor nanoparticles or quantum dots, where the particle size and size distribution are critical in defining the 275 optical and electronic properties and stability of the desired product. ${ }^{67}$ Both continuous and segmented flow microfluidic reactors have been shown to be of use in the intelligent synthesis of nanoparticles (Figure 6). As noted, microfluidic reactors are capable of controlling heat and mass transfer and can thus precisely define the process of particle nucleation and growth. Accordingly, particle populations with low 
reduced size distributions and uniform shape can be prepared by applying different synthetic methods

280 such as sol-gel chemistry, templated synthesis and microemulsions. ${ }^{68} \mathrm{~A}$ variety of nanoparticles (such as metallic, metal oxide, compound semiconductor, inorganic and polymeric) and functional biomaterials (microcapsules, microgels, vesicles and Janus microparticles) possessing well-defined sizes and shapes, have been synthesised within microfluidic systems. Table 2 in the Supplementary Information summarises many developments in the use of continuous and segmented flow microfluidic reactors for particle 285 synthesis and provides further information about typical functionalisation techniques and reactor materials. The intelligent synthesis of CdSe nanoparticles is shown in Figure 6. This work highlights the synergy between online monitoring of nanoparticle quality and the use of intelligent algorithms to change reaction conditions to enhance nanoparticle quality, without having to fully test all experimental parameters.

\section{Bottlenecks and Barriers}

There should always be a reason why a new technology is used to perform an established and functioning synthetic procedure. Admittedly, this reason may sometimes not be apparent before the application is

295 developed, but the technology should enable something that was not previously possible. The best applications for microfluidic reaction technology are in reactions or product lines where there is a major problem with the existing process, or where changing the scale of the reactor gives an overwhelming advantage. In an exceedingly well-argued paper Jensen and co-workers set out conditions where the use of microfluidic reaction technology makes sense..$^{18}$ The main condition is that the rate of reaction should

300 be rapid compared to the rate of mass transfer. Similarly, where the rate of heat transfer from the reaction is a limiting factor in a process, the advantages of microfluidic reactors are clear. Conversely, it can be argued that for reactions dominated by slow reaction rates, the advantages of microfluidic reactors are less apparent. Put simply, if there is no obvious constraint with a process as run on current technology, then microfluidic reactors have little applicability. 
During the development of microfluidic reaction technology there have been several areas of research that have turned out, with the benefit of hindsight, to be blind alleys. This is not to say that the research has no application, just that its utility in reaction technology is at best problematic. Chief among these redundant research areas is electroosmotic flow (EOF) pumping. ${ }^{69,70}$ EOF may have appeared attractive at

310 first sight because it allowed flat-profile fluid motivation through small channels under the control of simple electric currents. Needless to say, for automation this would be ideal. However, it is difficult to imagine a scaled system for EOF production-scale chemistry, as the highest flow rate through each channel is limited to around $5 \mu \mathrm{L} / \mathrm{min}$. Although it can be argued that EOF systems could be used for gathering reaction information, even this is doubtful since EOF is not a universal pumping system because

315 it affects the distribution of charged species within the reaction volume due to their electrophoretic mobilities. Given that the vast majority of reactions undertaken involve some ionic component and given the difficulties of scaling EOF reactors, this essentially means that information gathered in EOF devices will never be applicable on the production scale. Another sterile area of microfluidic reaction research was the development of microwell-based reaction systems, exemplified by the Orchid Biocomputer. ${ }^{71}$ Whilst these

320 systems are not without champions for applications in biomedical science, ${ }^{72,73}$ as a chemical reaction format they add very little that cannot be learnt from a 96-well plate.

From a synthetic perspective, many early studies on microfluidic reaction technology focused on performing popular small molecule reactions, such as the Wittig or Suzuki reactions, ${ }^{74,75}$ which are well

325 characterised, well behaved and well understood in bulk scale systems. Whilst this answered the question as to whether any chemistry could work in a microfluidic channel, it was unlikely to provide a new methodology for those reactions. This highlights a lack of understanding between laboratory-based microfluidic research and industry, where academia tends to focus on the projects that will produce scientifically relevant results, whereas industry is guided by commercial advantage. However, since 330 microfluidic methods present a technology with many advantages attractive for industrial interests, 
academic research would certainly benefit from considering potential industrial applicability as a measure of research relevance. Academic chemistry tends to still use reactors based on 19th century paradigms and it therefore may be constructive to embrace the industrialisation of research as a way of updating current research methodology. With this in mind, we will now explore the current bottlenecks to the 335 widespread use of microfluidic reactor technology.

Microfluidic reactor material. It is imperative to choose the reactor material with the application in mind. Substrate requirements may include: resistance to high pressures and temperatures, solvent compatibility, optical clarity, impermeability to reagents, appropriate surface chemistry and ease of

340 integration of fluidic elements such as pumps and valves. ${ }^{76,77}$ Traditional chip materials used for reactor miniaturisation include polydimethylsiloxane (PDMS), glass, quartz and silicon. ${ }^{78}$ Glass or silicon substrates offer high chemical robustness but require relatively complex and expensive fabrication processes. ${ }^{79}$ Other materials that have been used for microfluidic reactor fabrication include a diversity of polymers such as polymethyacrylate (PMMA), polycarbonate, polyester, fluorinated polymers, cyclic olefin

345 copolymers and polycrystalline diamond. ${ }^{77,80-82}$ Industrial microfluidic reactors are normally created from materials such as Hastelloy and aluminium $\left(\right.$ Lonza $\left.^{30}\right)$, fluoropolymers such as perfluoroalkoxy (PFA) and polytetrafluoroethylene (PTFE) or stainless steel $\left(\right.$ Uniqsis $\left.^{31}\right)$. In academia, PDMS is the most utilised material for device fabrication due to the ease of prototyping and its low cost. However, it has recognised drawbacks when used for chemical synthesis. Most importantly, PDMS devices cannot sustain high

350 pressures and temperatures, can accommodate only a narrow range of chemical reactions (organic solvents swell or dissolve PDMS) $)^{83}$ and are permeable to liquids and gases. These problems have been subjected to some ingenious solutions, including the coating of PDMS channels with glass, ${ }^{84}$ though these suggest that an easier solution may be to simply use devices made from compatible materials from the start! The fact that many research groups solely use soft lithography in device manufacture may have

355 created a roadblock in the path of device research for microfluidic chemical synthesis that is difficult to reverse. Conversely, potential problems with the microfluidic reactor material have sometimes been used 
to synthetic advantage. For example, Kreutz et al. ${ }^{85}$ used the porosity of Teflon-PFA to mediate the transport of oxygen and methane gas into droplets containing different catalysts for the oxidation of methane to methanol (Figure 7) and Elvira et al. have used the porosity of fluorinated ethylene propylene

360 (FEP) microcapillary films to dose singlet oxygen into solvents for use in industrial scale, non-hazardous oxidations. $^{86}$

Multiphase reactions. The use of microfluidic reactors for multiphase processes (involving solids, gases, liquids and supercritical fluids) ${ }^{87}$ offers clear advantages over conventional methods (such as sparged 365 reactors or stirred vessels) due to the increased surface area-to-volume ratios. Specifically, the more intimate surface contact between the different phases translates into a reduction of undesired mass transport effects and shows improved selectivity in heterogeneous catalytic studies of multiphasic (gasliquid-solid) reaction systems in microfabricated packed-bed reactors. ${ }^{88}$ This also results in superior heat and mass transfer within the system and controlled mixing of reagent streams, improving the reaction 370 rates, yields, and/or selectivities of a number of gas-liquid and gas-liquid-solid reactions. ${ }^{89,90}$ Despite these attractive features, microfluidic reactors are not well suited to handling solids, either as reaction byproducts or as reagents. Issues such as clogging and how to introduce solids into the reactor need to be addressed properly. ${ }^{67}$ This aspect has been assessed recently, ${ }^{91}$ and innovative solutions have been developed, such as the use of droplet-based microfluidics ${ }^{92}$ and the application of ultrasound through an 375 integrated piezoactuator on a Teflon-based microfluidic reactor. ${ }^{93}$ This also holds true from an industrial and ecological perspective; the potential major detriment to the use of microfluidic reactors in industry is the lifetime of the reactor due to clogging or breakage. ${ }^{56}$ Finally, the use of solid supported reagents as a method for performing complex reaction sequences has been demonstrated in several elegant systems. ${ }^{94}$ This approach has clear advantages in terms of product purity but suffers from the batch-wise nature of solid supported reagents, making it difficult to envisage continuous production. 
Reactor format. The format of a reactor is as important to the success of its application as any other factor. It is easy for researchers to become used to designing in one way (such as small rectangular chips) and to apply this format to any given project out of habit, without considering the demands of heat flow, 385 port access, reactor functionality and reactor mounting. A good example is the very efficient parallel laminar micromixer designed by Bessoth and co-workers. ${ }^{95}$ The internal architecture of this design was outstandingly efficient, but the external architecture was designed with the ports so close together that a complex interface system was required to access them. ${ }^{96}$ Similarly, most reported continuous flow polymerase chain reaction (PCR) systems, which require precise heat flow control, ignore the laws of 390 thermodynamics ${ }^{97}$ or the fact that rectangular shapes cool asymmetrically. ${ }^{98}$ This is not to say that the systems do not work, but design flaws mean excess energy must be used to maintain optimum operating conditions. Another puzzling aspect of microfluidic synthesis research is how often delay (incubation) channels are fabricated laboriously from planar substrates, when in many cases a simple capillary would perform the same function. ${ }^{85,99}$ In simple terms, the format selected should be the one that makes most

395 sense given the constraints of the system under study. This does not specifically mean that one reactor must be designed for each new reaction studied because reactions can be classified by constraints. It does however mean that one reactor format should not be used for all reactions without consideration of their requirements.

400 Scale. As shown in Figure 5, size is important. The microscale offers great advantages due to its unique properties, but so do the milli- and the macroscale. ${ }^{100}$ Research into the microscale properties of fluids can embark on projects designed to replicate macroscale phenomena (such as distillation, ${ }^{101,102}$ centrifugation, ${ }^{103,104}$ phase separation ${ }^{105}$ ) on the microscale. Whilst this can be a scientifically interesting exercise, it is of doubtful application: if something works well at a larger scale it may be best to operate at 405 this scale to exploit these advantages. The classic examples here would be any system employing bulk forces such as gravity, or negative or positive buoyancy (in droplet separations for example) to operate. As buoyant forces scale with the cube of droplet radius, this mode of separation becomes progressively less 
efficient as the scale decreases. ${ }^{106}$ Whilst large-scale systems cannot always be employed due to sample constraints, it should be axiomatic that a good designer always employs the scale most appropriate to the 410 problem at hand.

These barriers or bottlenecks to the widespread use of microfluidic reactor technology are applicable to the field in general. When considering microfluidic reactors for use in chemical synthesis there are also other important factors to take into account. Organic chemistry requires that microfluidic reactors be 415 resistant to solvents, acids, bases, oxidants and reductants, that they operate in temperature regimes between -78 and $300^{\circ} \mathrm{C}$, that mixing of reagents is efficient and versatile, that sufficient incubation is possible and that reaction quenching and, as a minimum, initial reaction clean-up such as liquid-liquid extraction, are also achievable within the microfluidic reactor. As we have shown throughout this review and specifically in the catalogue of chemical syntheses provided in Table 1 in the Supplementary

420 Information, microfluidic reactors provide ideal environments especially for reactions with hazardous reagents and/or products, air sensitive reactions (lithiations, metalations) and for explosive chemistry (azides). However, there are additional interesting areas where the use of microfluidic reactors can potentially have great impact, such as multistep synthesis, ${ }^{61}$ selective selection of reaction intermediates, ${ }^{107}$ mechanistic studies ${ }^{86}$ and high-throughput chemistry. ${ }^{108,109}$

\section{Future Challenges}

In 1900 David Hilbert proposed a series of 23 mathematical problems which set out the challenges, as he saw them, for modern mathematics. ${ }^{110}$ This lecture, translated into many languages, stimulated 430 mathematical research for decades. Our intention here is to attempt, in a far less ambitious way, the same feat for microfluidic research by defining challenges intended to enable the full potential of microfluidic reaction technology to be realised. Much work is already being done towards the completion 
of this list, and some items may be in the process of being accomplished. It is our hope is that when this list has been accomplished, microfluidic reaction technology will have completed the move from research 435 subject to standard ubiquitous technique.

\section{Compatible analytical technology}

As described above, many analytical techniques have been, and are being, interfaced with microfluidic reaction technology. However, very few of these techniques are able to operate with the same sampling

440 frequency as the devices themselves. The rate at which samples can be generated in a microfluidic device essentially depends only on the accessible flow ramping rates of the pumps used, whereas the sampling rate of HPLC, for example, is several orders of magnitude below this. Whilst electrospray ionization mass spectrometry (ESI-MS) and flow-cell based techniques can operate at more useful sampling rates, these techniques can struggle to resolve complex mixtures of similar molecules. We would deem this challenge

445 to have been completed when a microfluidic synthesis system is able to synthesise and resolve in analysis positional isomers of small drug-like molecules with a sampling rate measured in hundreds of Hertz.

\section{Truly different phases}

When multiple fluid phases are used in microfluidic reaction systems, the fluids are often quite similar in 450 terms of their viscosity, flow behaviour, compressibility etc. There will understandably be serious potential advantages in operating systems with radically different viscosities, for example, or with combinations of Newtonian and non-Newtonian fluids, or the full exploitation of gas-liquid flows such as aerosols. We would deem this challenge to have been completed if a microfluidic system capable of stably processing two flowing phases with a viscosity difference greater than $10^{4} \mathrm{P}$ is created. 


\section{Full use of surfaces}

Whilst much excellent work has been done on the use of surface patterning, solid supported reagents and

460 adsorption within microfluidic devices, ${ }^{111}$ the full use of surfaces remains a minor component of reactor design and use for the greater microfluidic community. Given that surfaces dominate the reaction space within microfluidic environments, this is an obvious oversight. We would deem this challenge to have been completed when chemically active, non-labile, patterned surface modification of reactor surfaces for operating periods in excess of 12 hours have been demonstrated on glass, silicon dioxide, polymer and 465 metal microfluidic reactors.

\section{Processing of Solids}

Most reviews on microfluidic reaction technology mention that reactors are unsuited to the processing of solids. Although work in micro- and nano-particle formation has shown this not to be entirely true, this

470 perception is generally accepted. It is plain that solids larger than the channel dimensions cannot be processed through microfluidic devices, but it is not necessarily true to say the same of those with dimensions smaller than the channels. We would deem this challenge to have been completed when a microfluidic system capable of processing two different powders (one cohesive and one non-cohesive) in suspension, where the powder granule size is between 0.1 and 0.01 channel widths, has been

475 demonstrated. Processing in this case means dispensing separately or in any desired, perfectly mixed, combination.

\section{Fully selective multi-product synthesis}

Microreaction systems are capable of great chemical selectivity, but this is normally exhibited in terms of 480 the reduction of the formation of a by-product from a specific mixture. The finest control over a reaction would be the ability to select any desired product from the reaction and to make it exclusively. We would 
deem this challenge to have been completed when a microfluidic reaction system has been demonstrated which can produce every possible product from a given reaction in greater than $90 \%$ selectivity.

\section{Reaction phase space mapping}

Reaction optimisation via robotic or parallel systems utilises design of experiments to minimise the total number of experiments that need to be performed to find optima within the reaction phase space. This minimisation is necessary because to accurately map phase space for a reaction in high density is extremely costly and time consuming. Microfluidic reaction systems can potentially read, register and

490 measure data far faster than existing systems, opening up the possibility of true phase space mapping. We would deem this challenge to have been completed when the entire set of product outcomes (conversions, yields, by-product yields) for an organic synthetic reaction has been mapped for a set of conditions consisting of starting material mix ratios from 0 to 1 in 0.01 steps, reagent concentrations from 0 to $15 \%$ $(\mathrm{w} / \mathrm{v}$ or $\mathrm{v} / \mathrm{v})$ in $0.01 \%$ steps and temperature from $-18^{\circ} \mathrm{C}$ to the 1 atmosphere boiling point of the solvent 495 employed in $2^{\circ} \mathrm{C}$ steps.

\section{Discovery chemistry}

Finally, it is motivating to consider that to date no new or unique molecule of proposed chemical utility has been synthesised within a microfluidic reactor. Given that several reactions that are not feasible on

500 the bench or pilot scale have been shown to be easily accomplished within a microfluidic environment, it seems bizarre that no new molecule has been synthesised in a microfluidic reactor. We would deem this challenge to have been completed when a novel molecule of chemical or biological utility is synthesised using only microfluidic technology for the major reactive steps, and where one of those steps is feasible only within a microfluidic environment.

505 
Microfluidic reaction systems have come a long way towards fulfilling most of the predictions made for them early on. These systems are slowly being integrated into mainstream synthesis and discovery laboratories as useful techniques for demanding situations, and this recognition continues to grow. We hope that these challenges will inspire future endeavour in the way that David Hilbert's original paper encouraged mathematical research. 
The authors declare no competing financial interests.

\section{Author contributions}

515 K.S.E., R.C.R.W. and A.J.dM developed the concept, discussed the results and implications and edited and commented on the manuscript at all stages; K.S.E., R.C.R.W and X.C.S researched and wrote the manuscript and K.S.E. edited the Supplementary Information.

\section{Corresponding authors}

Department of Chemistry and Applied Biosciences, Institute for Chemical and Bioengineering, ETH Zürich, Wolfgang-Pauli Strasse 10, Zürich, 8093, Switzerland. e-mail: katherine.elvira@chem.ethz.ch and andrew.demello@chem.ethz.ch.

\section{Acknowledgements}

The authors acknowledge loannis Lignos and Anand Rane for the literature research shown in Tables 1 and 2 in the Supplementary Information. 


\section{What is a microfluidic reactor?}

535 The performance of a chemical reaction within a microfluidic reactor should take advantage of the unique characteristics of fluid flow on the microscale. The advantages of downsizing are guaranteed when the characteristic dimension of a microfluidic reactor is below $500 \mu \mathrm{m}$; generally accepted as the definition of a microfluidic reactor. A more general and flexible definition for a microfluidic reactor is one that operates at Reynolds and thermal Péclet numbers below 250 and 1000 respectively ${ }^{\zeta}$. With this in mind, it is also possible to

540 define a transitional regime corresponding to reactors with a characteristic dimension between 500 and $1000 \mu \mathrm{m}$ where, dependent on the flow rate, some flow characteristics may be termed microfluidic. This regime overlaps the definition of "mesofluidic" reactors, which are generally considered to have characteristic dimensions between $500 \mu \mathrm{m}$ and a few millimetres. However, it is important to note that when microfluidic reactors have a characteristic dimension above $1 \mathrm{~mm}$ it is difficult to maintain a microfluidic regime without using extremely low 545 flow rates.

\section{Reynolds number}

The Reynolds number ( $\mathrm{Re})$ is a dimensionless number used to quantify the relative importance of inertial and viscous forces within a fluid.

$$
R e=\frac{\text { inertial forces }}{\text { viscous forces }}=\frac{\rho d v}{\mu}
$$

Re accounts for characteristics of both the fluid (density, $\rho$, and dynamic viscosity, $\mu$ ) and the environment in which the fluid is manipulated (fluid velocity, $v$, and characteristic dimension, $d$ ). Fluid flow in microfluidic devices is characterised by low Reynolds numbers (generally below 250) and is located in the laminar flow regime. When chemical reactions are performed in microfluidic reactors, low Re ensure that there is no turbulence and hence no 555 back-mixing within the reactor.

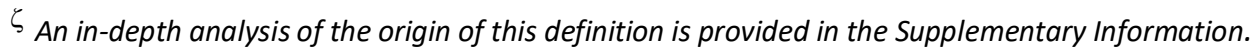




\section{Thermal Péclet number}

The thermal Péclet number $\left(P e_{L}\right)$ is a dimensionless number often used to characterise the microfluidic regime. $P e_{L}$ describes the ratio between heat transport due to advective and diffusive transport, i.e.

$$
P e_{L}=\frac{\text { advective heat transport }}{\text { diffusive heat transport }}=\frac{d v \rho C_{p}}{k}
$$

560 Here, $d$ is the channel diameter, $v$ is the fluid velocity, $\rho$ is the density of the fluid, $k$ is the thermal conductivity of the fluid and $C_{p}$ is its specific heat capacity. The thermal Péclet number is especially relevant for chemistry performed in a microfluidic environment since it describes the relative importance of heat transfer due to bulk fluid motion and conductive heat transfer. For example, in a microfluidic channel where heat is applied to the channel wall, the thermal Péclet number will define how rapidly heat is carried by the moving fluid versus how fast 565 it is transferred from the channel wall to the center of the fluid flow. 

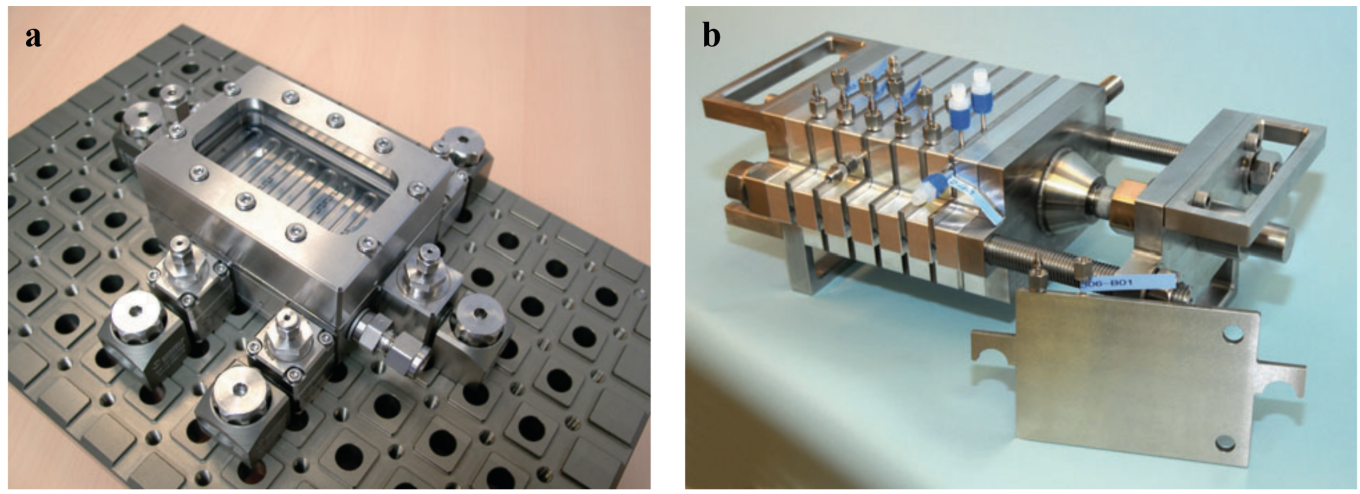

Figure 1 - Microfluidic reactors for industrial processes. a, The Lonza FlowPlate Lab microfluidic reactor can be used for laboratory-based experimental process development. The reactor incorporates a viewing window to facilitate detection and monitoring and was designed to enable facile scale out, and/or scale up to the milli-scale, for industrial production. $\mathbf{b}$, The Lonza FlowPlate A6 microfluidic reactor can be used for process development in the kilogram range. It was developed to take advantage of the benefits of microfluidic reactors (enhanced heat transfer, rapid mixing times, small volumes) and enables hazardous reactions to be performed in safe environments on an industrial scale. ${ }^{47}$ 


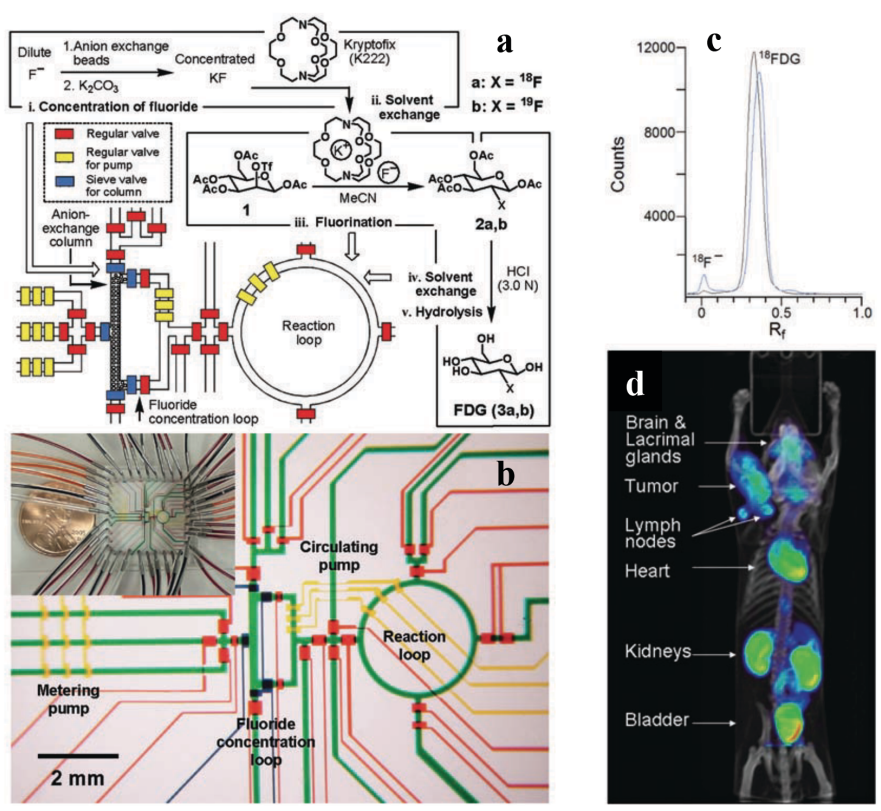

Figure 2 - Microfluidic reactors for integrated synthesis. On-chip, integrated synthesis of the imaging probe 2-deoxy-2[18 F]fluoro-D-glucose ([18F]FDG) with subsequent testing of the probe in mice. The multi-step synthesis was performed in a PDMS microfluidic reactor and is shown in steps (i) to (v) in a. The PDMS valve mechanism used to dose and extract reagents 605 and products is shown schematically in a, and as a photograph with reference to the complete device in b. Spectra for the produced [ $\left.{ }^{18} \mathrm{~F}\right] \mathrm{FDG}$ is shown in $\mathbf{c}$ and $\mathbf{d}$ shows a microPET/microCT image for the in vivo use of the [18F]FDG synthesised in the microfluidic reactor. ${ }^{21}$ 


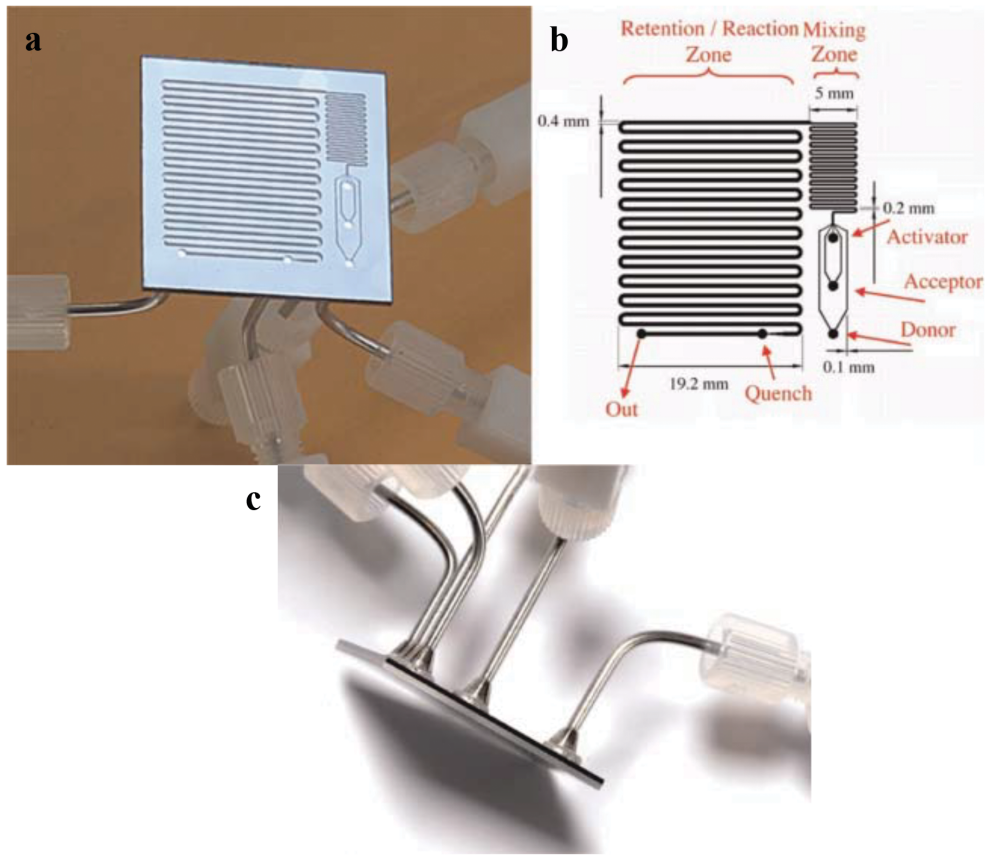

Figure 3 - Microfluidic reactors for reaction optimisation. Reaction optimisation was performed on a microfluidic reactor using

615 glycosylation reactions as an example. Reaction temperature, reagent concentration and residence time were varied and 44 different conditions were sampled to find the optimum reaction conditions. The silicon and Pyrex microfluidic reactor shown in a was used for the synthesis. The design $\mathbf{b}$ is simple in structure, with three inlets for glycosylating agent (donor), nucleophile (acceptor) and activator addition, a mixing zone and a reaction zone, with the quenchant being added towards the end. Connectors are soldered to the device as shown in c. ${ }^{112}$ 
a

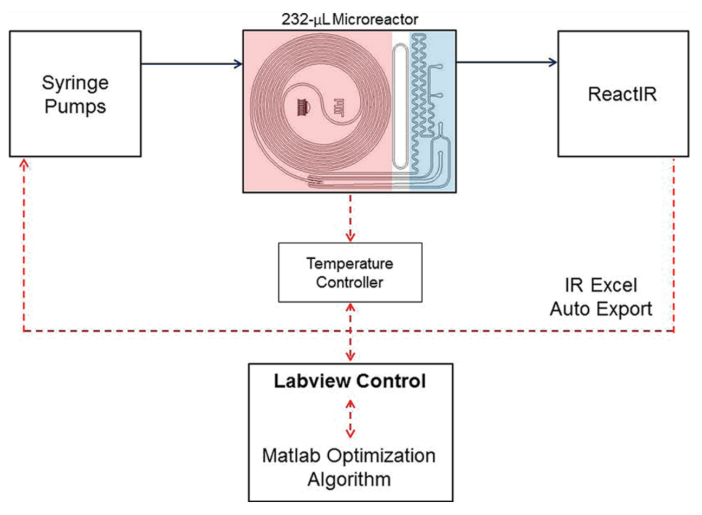

b
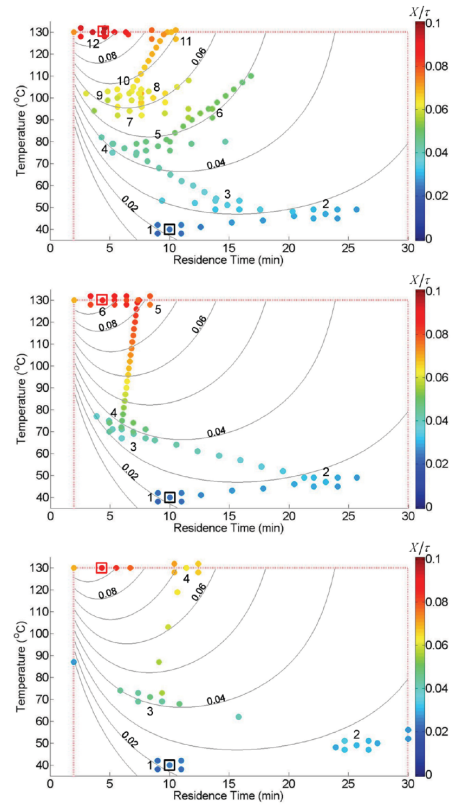

625 Figure 4 - Microfluidic reactors for reaction optimisation with continuous monitoring. Continuous online IR analysis of the product of a Paal-Knorr reaction allowed for reaction optimisation using several different multi-trajectory optimisation strategies. a, Experimental setup showing both fluid flow (solid lines) and data flow (dashed lines). $\mathbf{b}$, Three different methods were assessed (from top to bottom): steepest gradient, conjugate gradient and Armijo conjugate gradient. The colour scale shows the value of the objective function, $\mathrm{X} / \tau$, where $\mathrm{X}$ is the conversion fraction and $\tau$ is the residence time. The black box denotes the initial conditions in each case and the optimal conditions are highlighted by a red box. The Armijo conjugate gradient method is shown to be the most efficient. ${ }^{113}$ 


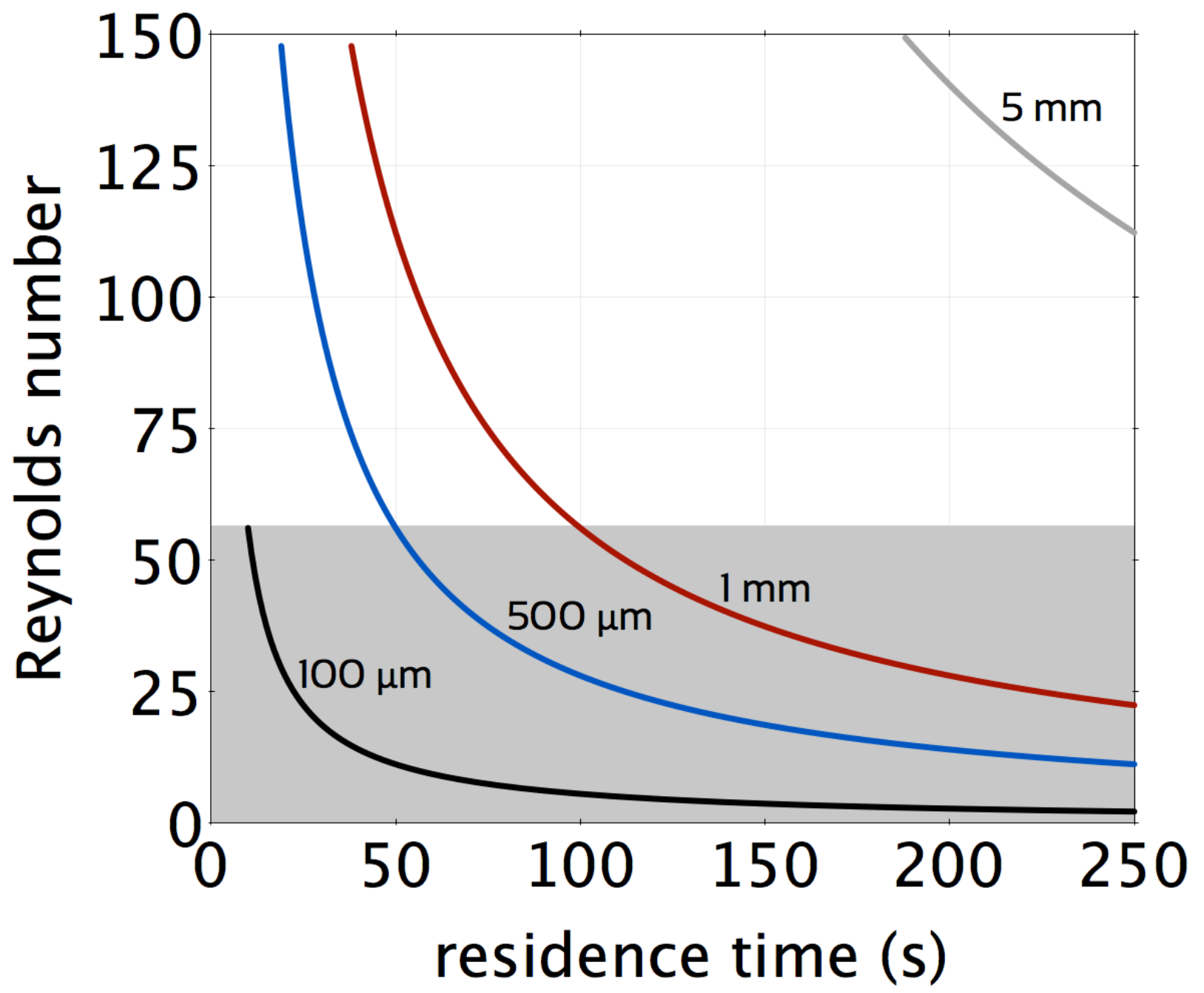

Figure 5 - Microfluidic reactor size considerations. A plot of Reynolds number versus residence time for microfluidic reactors of 5 metres in length and channel diameters ranging between $100 \mu \mathrm{m}$ and $5 \mathrm{~mm}$. Data show that, for a wide range of flow rates, both $500 \mu \mathrm{m}$ and $1 \mathrm{~mm}$ diameter microfluidic reactors operate in similar Reynolds number regimes to the classical (100 $\mu \mathrm{m}$ diameter) microfluidic reactors. It should be noted, however, that mixing, thermal diffusion and other representative microfluidic parameters scale non-linearly with the characteristic dimension. Additional information is provided in the Supplementary Information. 


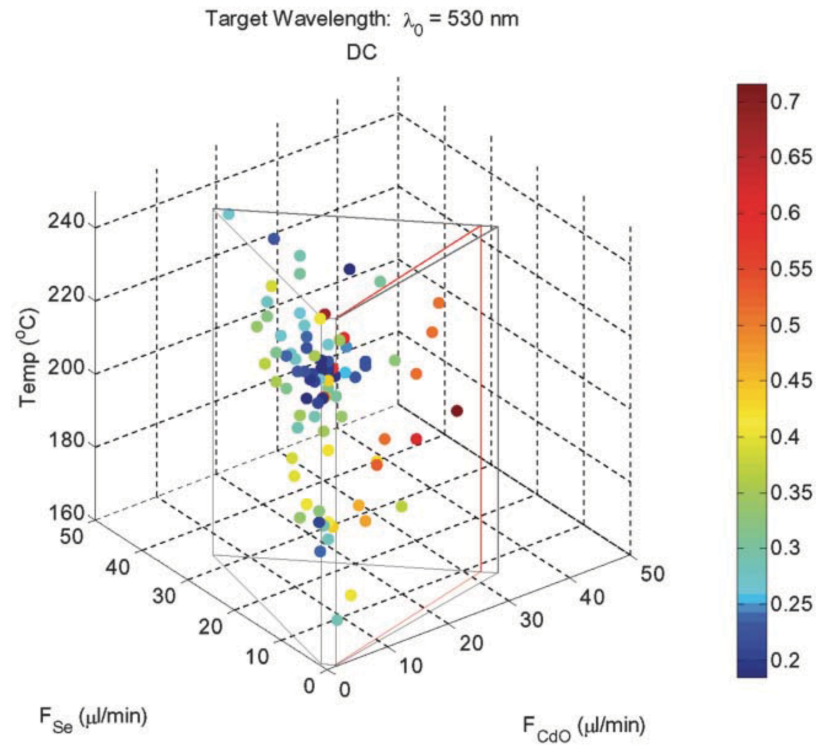

650

Figure 6 - Microfluidic reactors for intelligent synthesis. The intelligent synthesis of CdSe nanoparticles was achieved by in-line monitoring of the emission spectra of particles produced in a microfluidic reactor. A control algorithm was used to determine a 'dissatisfaction coefficient' as a measure of the quality of the particles with respect to a pre-set target. The algorithm then changed the reaction conditions to attempt to minimise this coefficient. In this graph, the target wavelength is $530 \mathrm{~nm}$ and the colour of each dot shows the value of the dissatisfaction coefficient for each experiment with respect to the flow rates of Se and $\mathrm{CdO}$ and the reaction temperature (the grey lines provide maximum possible values for each). ${ }^{108}$ 


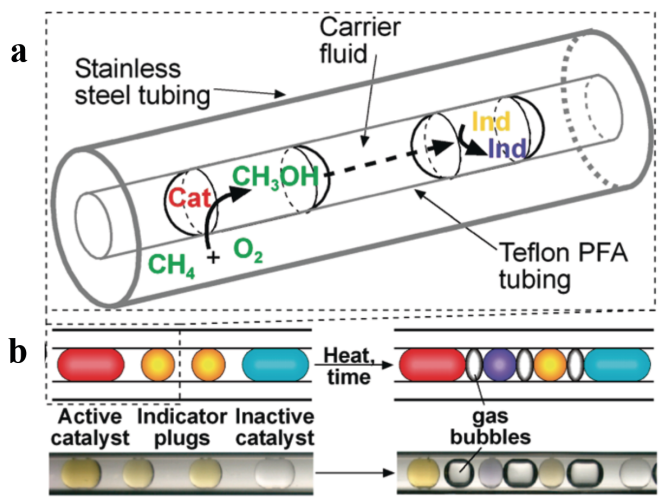

Figure 7 - Microfluidic reactors for discovery catalyst research. A microfluidic reactor and genetic algorithms were used to find new catalysts for the oxidation of methane. a, Hundreds of reaction conditions were assayed in droplets. The authors took advantage of the permeability of Teflon tubing to mediate gas (methane and oxygen) access to the catalyst-containing droplets. Droplets containing active catalysts were detected by the colour change in neighbouring indicator droplets, which only changed colour when the reaction product, methanol, leached from an adjacent droplet. $\mathbf{b}$, Schematic and photographs of the experiment in progress. Catalysts were separated by two indication plugs to ensure correct identification of active catalysts. Upon heating, methanol diffused through the fluorous continuous phase and caused a colour change from yellow to purple in an adjacent indication plug. ${ }^{85}$ 
Table of contents graphic and summary of article

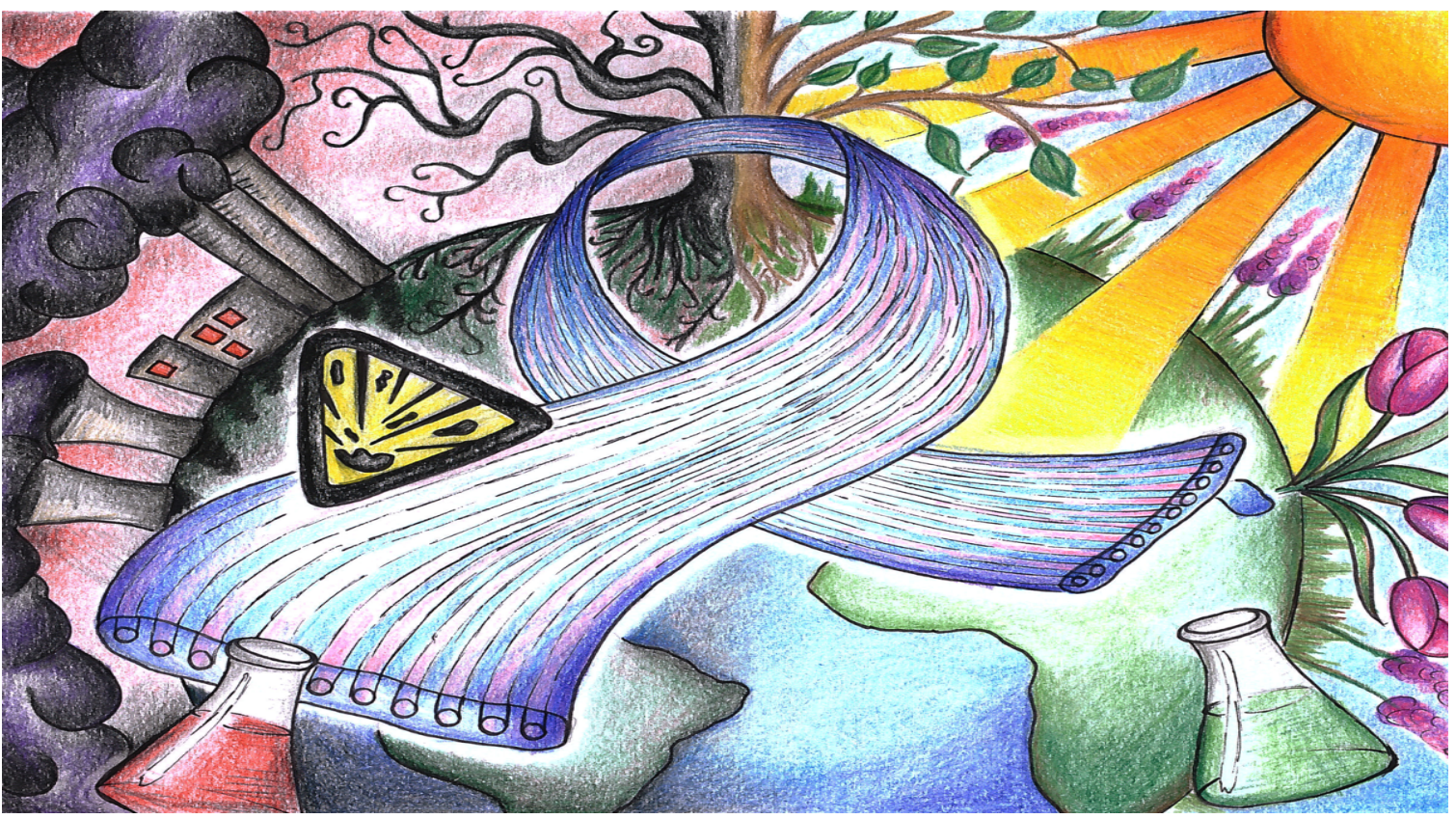

Microfluidic reactor technology has important advantages for synthetic chemistry. In this article we highlight important successes and failures in this field, provide an extensive summary of syntheses performed in microfluidic reactors, assess the current roadblocks hindering their

690 widespread use, and set out seven challenges that we hope will inspire future research. 
1 Philpot, J. S. L. The use of thin layers in electrophoretic separation. Trans. Faraday Soc. 35, 38-46, (1940).

2 Terry, S. C. A gas chromatography system fabricated on a silicon wafer using integrated circuit technology. Stanford Electronics Laboratories Technical Report 4603, 41, (1975).

7053 Manz, A., Graber, N. \& Widmer, H. M. Miniaturized total chemical analysis systems: A novel concept for chemical sensing. Sens. Actuators, B 1, 244-248, (1990).

4 Woolley, A. T. \& Mathies, R. A. Ultra-high-speed DNA fragment separations using microfabricated capillary array electrophoresis chips. Proc. Natl. Acad. Sci. U. S. A. 91, 11348-11352, (1994).

710 microreactor system. Lab Chip 2, 31-33, (2002).

6 Srinivasan, R. et al. Chemical performance and high temperature characterization of micromachined chemical reactors. Transducers 97, Int. Conf. Solid-State Sens. Actuators 1, 163-166, (1997).

7 Ehrfeld, W., Golbig, K., Hessel, V., Löwe, H. \& Richter, T. Characterization of mixing in micromixers by a test reaction: Single mixing units and mixer arrays. Ind. Eng. Chem. Res. 38, 1075-1082, (1999).

7158 Mitchell, M. C., Spikmans, V., Manz, A. \& de Mello, A. J. Microchip-based synthesis and total analysis systems ( $\mu$-SYNTAS): Chemical microprocessing for generation and analysis of compound libraries. J. Chem. Soc., Perkin Trans. 1, 514-518, (2001).

9 Schwalbe, T., Autze, V. \& Wille, G. Chemical synthesis in microreactors. Chimia 56, 636-646, (2002).

10 Goodell, J. R. et al. Development of an automated microfluidic reaction platform for multidimensional screening: Reaction discovery employing bicyclo[3.2.1] octanoid scaffolds. J. Org. Chem. 74, 6169-6180, (2009).

11 Zaborenko, N., Bedore, M. W., Jamison, T. F. \& Jensen, K. F. Kinetic and scale-up investigations of epoxide aminolysis in microreactors at high temperatures and pressures. Org. Process Res. Dev. 15, 131-139, (2010).

12 Shimoyama, A., Fujimoto, Y. \& Fukase, K. Stereoselective glycosylation of 3-deoxy-d-manno-2-octulosonic acid with batch and microfluidic methods. Synlett 2011, 2359-2362, (2011).

72513 Asai, T. et al. Switching reaction pathways of benzo[b]thiophen-3-yllithium and benzo[b]furan-3-yllithium based on highresolution residence-time and temperature control in a flow microreactor. Chem. Lett. 40, 393-395, (2011).

14 Pieber, B. \& Kappe, C. O. Direct aerobic oxidation of 2-benzylpyridines in a gas-liquid continuous-flow regime using propylene carbonate as a solvent. Green Chem. 15, 320-324, (2013).

15 Wiles, C., Watts, P. \& Haswell, S. J. Clean and selective oxidation of aromatic alcohols using silica-supported Jones' reagent

$730 \quad 16$ in a pressure-driven flow reactor. Tetrahedron Lett. 47, 5261-5264, (2006). 166, (2001).

17 Illg, T., Hessel, V., Lob, P. \& Schouten, J. C. Continuous synthesis of tert-butyl peroxypivalate using a single-channel microreactor equipped with orifices as emulsification units. ChemSusChem 4, 392-398, (2011).

73518 Hartman, R. L., McMullen, J. P. \& Jensen, K. F. Deciding whether to go with the flow: Evaluating the merits of flow reactors for synthesis. Angew. Chem., Int. Ed. 50, 7502-7519, (2011).

19 Saias, L., Autebert, J., Malaquin, L. \& Viovy, J.-L. Design, modeling and characterization of microfluidic architectures for high flow rate, small footprint microfluidic systems. Lab Chip 11, 822-832, (2011).

20 Ozdemir, M. R., Kosar, A., Demir, O., Ozenel, C. \& Bahcivan, O. Thermal-hydraulic, exergy and exergy-economic analysis of micro heat sinks at high flow rates. Proc. ASME Bienn. Conf. Eng. Syst. Des. Anal., 10th 5, 703-710, (2010).

21 Lee, C.-C. et al. Multistep synthesis of a radiolabeled imaging probe using integrated microfluidics. Science 310, 17931796, (2005).

22 Pelleter, J. \& Renaud, F. Facile, fast and safe process development of nitration and bromination reactions using continuous flow reactors. Org. Process Res. Dev. 13, 698-705, (2009).

74523 Norton, D. G., Wetzel, E. D. \& Vlachos, D. G. Fabrication of single-channel catalytic microburners: Effect of confinement on the oxidation of hydrogen/air mixtures. Ind. Eng. Chem. Res. 43, 4833-4840, (2004).

24 Geyer, K., Codee, J. D. \& Seeberger, P. H. Microreactors as tools for synthetic chemists - the chemists' round-bottomed flask of the 21st century? Chemistry 12, 8434-8442, (2006).

25 Haswell, S. J. \& Watts, P. Green chemistry: Synthesis in micro reactors. Green Chem. 5, 240-249, (2003).

75026 Amador, C., Gavriilidis, A. \& Angeli, P. Flow distribution in different microreactor scale-out geometries and the effect of manufacturing tolerances and channel blockage. Chem. Eng. J. 101, 379-390, (2004). 
27 Fletcher, P. \& Haswell, S. Downsizing synthesis. Chem. Br. 35, 38-41, (1999).

28 Steel, C. J., O'Brien, A. T., Luthra, S. K. \& Brady, F. Automated PET radiosyntheses using microfluidic devices. J. Labelled Compd. Radiopharm. 50, 308-311, (2007).

75529 McMullen, J. P., Stone, M. T., Buchwald, S. L. \& Jensen, K. F. An integrated microreactor system for self-optimization of a Heck reaction: From micro- to mesoscale flow systems. Angew. Chem., Int. Ed. 122, 7230-7234, (2010).

30 Kockmann, N., Gottsponer, M. \& Roberge, D. M. Scale-up concept of single-channel microreactors from process development to industrial production. Chem. Eng. J. 167, 718-726, (2011).

760 aminoethyl)acylamides through ring-opening/hydrogenation of oxazolines. Chem.-Eur. J. 17, 13146-13150, (2011).

32 Razzaq, T. \& Kappe, C. O. Continuous flow organic synthesis under high-temperature/pressure conditions. Chem.-Asian J. 5, 1274-1289, (2010).

33 Nobis, M. \& Roberge, D. Mastering ozonolysis: Production from laboratory to ton scale in continuous flow. Chimica Oggi 29, 56-58, (2011).

76534 Kockmann, N. \& Roberge, D. M. Scale-up concept for modular microstructured reactors based on mixing, heat transfer, and reactor safety. Chem. Eng. Process. 50, 1017-1026, (2011).

35 Pennemann, H., Watts, P., Haswell, S. J., Hessel, V. \& Löwe, H. Benchmarking of microreactor applications. Org. Process Res. Dev. 8, 422-439, (2004).

770

36 Hessel, V., Knobloch, C. \& Löwe, H. Review on patents in microreactor and micro process engineering. Recent Pat. Chem. Eng. 1, 1-16, (2008).

37 Dencic, I. et al. Recent changes in patenting behavior in microprocess technology and its possible use for gas-liquid reactions and the oxidation of glucose. ChemSusChem 5, 232-245, (2012).

38 Kockmann, N. \& Gottsponer, M. Heat transfer limitations of gas-liquid exothermic reactions in microchannels. ASME 2010, 3rd Jt. US-Eur. Fluids Eng. Summer Meet., 8th Int. Conf. Nanochannels, Microchannels, Minichannels, 193-199, (2010).

77539 Ducry, L. \& Roberge, D. M. Controlled autocatalytic nitration of phenol in a microreactor. Angew. Chem., Int. Ed. 44, 79727975, (2005).

40 Barthe, P. et al. Continuous multi-injection reactor for multipurpose production - part I. Chem. Eng. Technol. 31, 11461154, (2008).

41 Kockmann, N. \& Roberge, D. M. Transitional flow and related transport phenomena in curved microchannels. Heat

$780 \quad 42$ Transfer Eng. 32, 595-608, (2011). microreactors: Simplified designs of micromixers. Chem. Eng. Process. 50, 1069-1075, (2011).

43 Kashid, M. N. \& Kiwi-Minsker, L. Microstructured reactors for multiphase reactions: State of the art. Ind. Eng. Chem. Res. 48, 6465-6485, (2009).

78544 Roberge, D. M. et al. Microreactor technology and continuous processes in the fine chemical and pharmaceutical industry: Is the revolution underway? Org. Process Res. Dev. 12, 905-910, (2008).

45 Hessel, V., Renken, A., Schouten, J. C. \& Yoshida, J. Micro process engineering : A comprehensive handbook. Volume 2: Devices, reactions and applications. (Wiley- $\mathrm{VCH}, 2009$ ). $\begin{aligned} 46 & \begin{array}{l}\text { Tonkovich, A. et al. Microchannel technology scale-up to commercial capacity. Chem. Eng. Res. Des. 83, 634-639, (2005). } \\ \text { Roberge, D. Lonza - hazardous flow chemistry for streamlined large scale synthesis. Green Process. Synth. 1, 129-130, } \\ \text { (2012). }\end{array}\end{aligned}$

48 Yoshida, J. Flash chemistry: Fast organic synthesis in microsystems. (John Wiley \& Sons, Ltd., 2008).

49 Kockmann, N., Gottsponer, M., Zimmermann, B. \& Roberge, D. M. Enabling continuous-flow chemistry in microstructured devices for pharmaceutical and fine-chemical production. Chem.-Eur. J. 14, 7470-7477, (2008).

79550 Delville, M. M. E. et al. Continuous flow azide formation: Optimization and scale-up. Chem. Eng. J. 167, 556-559, (2011).

51 Alfa laval. Accessed March 2013, www.alfalaval.com/campaigns/labplatereactor/Pages/default.aspx.

52 Corning. Accessed March 2013, www.corning.com/products_services/new_business_development/reactors/index.aspx.

53 Microinnova. Accessed March 2013, www.microinnova.com/.

54 Chemtrix. Accessed March 2013, www.chemtrix.com.

80055 Futurechemistry. Accessed March 2013, www.futurechemistry.com.

56 Kralisch, D. \& Kreisel, G. Assessment of the ecological potential of microreaction technology. Chem. Eng. Sci. 62, 10941100, (2007).

57 Roberge, D. M. et al. Development of an industrial multi-injection microreactor for fast and exothermic reactions - part II. Chem. Eng. Technol. 31, 1155-1161, (2008).

80558 Schwalbe, T., Kursawe, A. \& Sommer, J. Application report on operating cellular process chemistry plants in fine chemical and contract manufacturing industries. Chem. Eng. Technol. 28, 408-419, (2005).

59 D. Chambers, R. \& C. H. Spink, R. Microreactors for elemental fluorine. Chem. Commun., 883-884, (1999).

60 Chambers, R. D., Holling, D., Spink, R. C. H. \& Sandford, G. Elemental fluorine part 13. Gas-liquid thin film microreactors for selective direct fluorination. Lab Chip 1, 132-137, (2001). 
81061 Rensch, C. et al. Microfluidic reactor geometries for radiolysis reduction in radiopharmaceuticals. Appl. Radiat. Isot. 70, 1691-1697, (2012).

62 Watts, P., Pascali, G. \& Salvadori, P. Positron emission tomography radiosynthesis in microreactors. J. Flow Chem. 2, 3742, (2012).

815 Synlett, 2382-2391, (2009).

$64 \mathrm{Illg}, \mathrm{T}$., Löb, P. \& Hessel, V. Flow chemistry using milli- and microstructured reactors - from conventional to novel process windows. Bioorg. Med. Chem. 18, 3707-3719, (2010).

65 Brivio, M., Verboom, W. \& Reinhoudt, D. N. Miniaturized continuous flow reaction vessels: Influence on chemical reactions. Lab Chip 6, 329-344, (2006).

82066 Koch, K. et al. Optimizing the deprotection of the amine protecting $p$-methoxyphenyl group in an automated microreactor platform. Org. Process Res. Dev. 13, 1003-1006, (2009).

67 McMullen, J. P. \& Jensen, K. F. Integrated microreactors for reaction automation: New approaches to reaction development. Annu. Rev. Anal. Chem. 3, 19-42, (2010).

82568 mesoporous silica microspheres. Langmuir 24, 658-661, (2008).

69 Doku, G. N., Haswell, S. J., McCreedy, T. \& Greenway, G. M. Electric field-induced mobilisation of multiphase solution systems based on the nitration of benzene in a micro reactor. Analyst 126, 14-20, (2001).

70 Watts, P., Wiles, C., Haswell, S. J., Pombo-Villar, E. \& Styring, P. The synthesis of peptides using micro reactors. Chem. Commun., 990-991, (2001).

83071 DeWitt, S. H. Microreactors for chemical synthesis. Curr. Opin. Chem. Biol. 3, 350-356, (1999).

72 Hou, F. H. et al. Development of a microplate reader compatible microfluidic chip for ELISA. Biomed. Microdevices 14, 729-737, (2012).

73 Conant, C. G. et al. Well plate microfluidic system for investigation of dynamic platelet behavior under variable shear loads. Biotechnol. Bioeng. 108, 2978-2987, (2011).

83574 Skelton, V. et al. The preparation of a series of nitrostilbene ester compounds using micro reactor technology. Analyst 126, 7-10, (2001).

75 Basheer, C., Shahitha, F., Hussain, J., Lee, H. K. \& Valiyaveettil, S. Design of a capillary-microreactor for efficient Suzuki coupling reactions. Tetrahedron Lett. 45, 7297-7300, (2004).

76 Whitesides, G. M. The origins and the future of microfluidics. Nature 442, 368-373, (2006).

84077 Gomez, F. A. Microfluidics in protein chromatography. Methods Mol. Biol. 681, 137-150, (2011).

78 Jensen, K. F. Silicon-based microchemical systems: Characteristics and applications. MRS Bulletin 31, 101-107, (2006).

79 Kim, D.-P. Lab-on-a-chip systems for microchemical applications. J. Anal. Sci. Technol. 2, 146-149, (2011).

80 Snyder, D. A. et al. Modular microreaction systems for homogeneously and heterogeneously catalyzed chemical synthesis. Helv. Chim. Acta 88, 1-9, (2005).

84581 Begolo, S., Colas, G., Viovy, J.-L. \& Malaquin, L. New family of fluorinated polymer chips for droplet and organic solvent microfluidics. Lab Chip 11, 508-512, (2011).

82 Rolland, J. P., Van Dam, R. M., Schorzman, D. A., Quake, S. R. \& DeSimone, J. M. Solvent-resistant photocurable "liquid Teflon" for microfluidic device fabrication. J. Am. Chem. Soc. 126, 2322-2323, (2004).

$850 \quad$ Chem. 75, 6544-6554, (2003).

84 Abate, A. R., Lee, D., Do, T., Holtze, C. \& Weitz, D. A. Glass coating for PDMS microfluidic channels by sol-gel methods. Lab Chip 8, 516-518, (2008).

85 Kreutz, J. E. et al. Evolution of catalysts directed by genetic algorithms in a plug-based microfluidic device tested with oxidation of methane by oxygen. J. Am. Chem. Soc. 132, 3128-3132, (2010).

85586 Elvira, K. S., Wootton, R. C. R., Reis, N. M., Mackley, M. R. \& deMello, A. J. Through-wall mass transport as a modality for safe generation of singlet oxygen in continuous flows. ACS Sustainable Chem. Eng. 1, 209-213, (2013).

87 Marre, S., Roig, Y. \& Aymonier, C. Supercritical microfluidics: Opportunities in flow-through chemistry and materials science. J. Supercrit. Fluids 66, 251-264, (2012).

Liu, X., Unal, B. \& Jensen, K. F. Heterogeneous catalysis with continuous flow microreactors. Catal. Sci. Technol. 2, 21342138, (2012).

89 Miller, P. W. et al. Rapid multiphase carbonylation reactions by using a microtube reactor: Applications in positron emission tomography 11C radiolabeling. Angew. Chem., Int. Ed. 119, 2933-2936, (2007).

90 Kobayashi, J. et al. A microfluidic device for conducting gas-liquid-solid hydrogenation reactions. Science 304, 1305-1308, (2004).

86591 Hartman, R. L. Managing solids in microreactors for the upstream continuous processing of fine chemicals. Org. Process Res. Dev. 16, 870-887, (2012). 
92 Frenz, L. et al. Droplet-based microreactors for the synthesis of magnetic iron oxide nanoparticles. Angew. Chem., Int. Ed. 47, 6817-6820, (2008).

93 Kuhn, S., Noel, T., Gu, L., Heider, P. L. \& Jensen, K. F. A Teflon microreactor with integrated piezoelectric actuator to handle solid forming reactions. Lab Chip 11, 2488-2492, (2011).

94 Baxendale, I. R., Ley, S. V., Mansfield, A. C. \& Smith, C. D. Multistep synthesis using modular flow reactors: BestmannOhira reagent for the formation of alkynes and triazole. Angew. Chem., Int. Ed. 48, 4017-4021, (2009).

95 Bessoth, F. G., deMello, A. J. \& Manz, A. Microstructure for efficient continuous flow mixing. Anal. Commun. 36, 213-215, (1999).

87596 Nittis, V., Fortt, R., Legge, C. H. \& de Mello, A. A high-pressure interconnect for chemical microsystem applications. Lab Chip 1, 148-152, (2001).

97 Chabert, M., Dorfman, K. D., de Cremoux, P., Roeraade, J. \& Viovy, J.-L. Automated microdroplet platform for sample manipulation and polymerase chain reaction. Anal. Chem. 78, 7722-7728, (2006).

880

98 Kopp, M. U., de Mello, A. J. \& Manz, A. Chemical amplification: Continuous-flow PCR on a chip. Science 280, 1046-1048, (1998).

99 Polyzos, A., O'Brien, M., Petersen, T. P., Baxendale, I. R. \& Ley, S. V. The continuous-flow synthesis of carboxylic acids using $\mathrm{CO}_{2}$ in a tube-in-tube gas permeable membrane reactor. Angew. Chem., Int. Ed. 50, 1190-1193, (2011).

100 Hartman, R. L. \& Jensen, K. F. Microchemical systems for continuous-flow synthesis. Lab Chip 9, 2495-2507, (2009).

101 Hartman, R. L., Sahoo, H. R., Yen, B. C. \& Jensen, K. F. Distillation in microchemical systems using capillary forces and segmented flow. Lab Chip 9, 1843-1849, (2009).

102 Wootton, R. C. R. \& De Mello, A. J. Continuous laminar evaporation: Micron-scale distillation. Chem. Commun., 266-267, (2004).

103 Lee, J. H. et al. Microfluidic centrifuge of nano-particles using rotating flow in a microchamber. Sens. Actuators, B 132, 525-530, (2008).

890104 Kang, J. Y., Cho, H. S., Kwak, S. M., Yoon, D. S. \& Kim, T. S. Novel particle separation using spiral channel and centrifugal force for plasma preparation from whole blood. Micro Total Anal. Syst. 2004, Proc. HTAS 2004 Symp., 8th 1, 614-616, (2004).

105 Kralj, J. G., Sahoo, H. R. \& Jensen, K. F. Integrated continuous microfluidic liquid-liquid extraction. Lab Chip 7, 256-263, (2007).

895106 Mason, T. G., Wilking, J. N., Meleson, K., Chang, C. B. \& Graves, S. M. Nanoemulsions: Formation, structure, and physical properties. J. Phys.: Condens. Matter 18, R635-R666, (2006).

107 Mitchell, M. C., Spikmans, V. \& deMello, A. J. Microchip-based synthesis and analysis: Control of multicomponent reaction products and intermediates. Analyst 126, 24-27, (2001).

108 Krishnadasan, S., Brown, R. J. C., deMello, A. J. \& deMello, J. C. Intelligent routes to the controlled synthesis of nanoparticles. Lab Chip 7, 1434-1441, (2007).

109 Garcia-Egido, E., Spikmans, V., Wong, S. Y. F. \& Warrington, B. H. Synthesis and analysis of combinatorial libraries performed in an automated micro reactor system. Lab Chip 3, 73-76, (2003).

110 Hilbert, D. Mathematical problems. Bull. Amer. Math. Soc. 8, 437-479, (1902).

111 Wootton, R. C. R. \& deMello, A. J. A one-step protocol for the chemical derivatisation of glass microfluidic devices. Lab Chip 6, 471-473, (2006).

112 Ratner, D. M. et al. Microreactor-based reaction optimization in organic chemistry - glycosylation as a challenge. Chem. Commun., 578-580, (2005).

113 Moore, J. S. \& Jensen, K. F. Automated multitrajectory method for reaction optimization in a microfluidic system using online IR analysis. Org. Process Res. Dev. 16, 1409-1415, (2012). 